INGENIERÍA DE SISTEMAS

\title{
Un detector de colisiones jerárquico basado en esferas exteriores e interiores
}

SYSTEM ENGINEERING

\section{A hierarchical collision detector based on outer and inner spheres}

\author{
Olmedo Arcila*§, Simena Dinas**, José M. Bañón** \\ *Facultad de Ingeniería, Universidad Autónoma de Occidente, Cali, Colombia \\ **Escuela de Ingeniería de Sistemas y Computación, Universidad del Valle, Cali, Colombia \\ §oarcila@uao.edu.co,simena.dinas@correounivalle.edu.co,jose.banon@correounivalle.edu.co
}

(Recibido: Marzo 6 de 2009 -Aceptado: Noviembre 15 de 2011 -Versión Final: Mayo 15 de 2012)

\begin{abstract}
Resumen
En este artículo se propone un modelo para detección de colisiones basado en doble representación esférica jerárquica: una representación en esferas exteriores cuyo objetivo es detectar la no intersección y una representación en esferas interiores para mejorar el desempeño del detector de colisiones. El algoritmo fue implementado y se realizaron varias pruebas con poliedros convexos así como también se hizo especial énfasis en las colisiones entre las esferas interiores. El resultado principal es que mientras las esferas exteriores sirven para detectar la ausencia de detecciones, las esferas interiores son más eficientes para detectar la existencia de colisiones y resultan útiles para acelerar el proceso de la detección de colisiones. El uso de esferas interiores mejora el desempeño general del algoritmo porque reduce el número de pruebas de intersección entre las caras de los poliedros.
\end{abstract}

Palabras Claves:Detección de colisiones, Representación esférica, Poliedro convexo.

\begin{abstract}
A model for collision detection based on double hierarchical spherical representation is proposed in this paper: an outer spherical representation for detecting no-intersections and an inner spherical representation in order to improve the performance of the collision detector. The algorithm has been implemented. Several tests were conducted with convex polyhedral and the performance of the algorithm was evaluated in terms of the collision between inner spheres. The experimental results show that outer spheres have been useful to determine lack of intersection, whereas inner spheres are more efficient for detecting existence of intersection and for speeding up the process of detecting collision. Using inner spheres improve the general performance because it reduces the number of intersection tests between the faces of the polyhedral.
\end{abstract}

Keywords: Collision detection, Spherical representation, Convexpolyhedron. 


\section{Introducción}

El problema de la detección de colisiones admite diferentes formulaciones dependiendo de la información que se requiera sobre la posición relativa entre varios objetos (García-Alonso, et al., 1994). Es un problema que depende enormemente de la aplicación. En su forma más general y básica es un problema de decisión: dado un conjunto de objetos en el espacio, determinar los pares de objetos que están en contacto. Los objetos pueden estar en movimiento o en reposo, en este último caso se habla de la detección de intersecciones. El problema de la detección de colisiones está íntimamente relacionado con el problema del cálculo de proximidad, el cual busca determinar la mínima distancia euclídea que separa dos objetos en el espacio, si los objetos están en contacto se busca determinar la distancia de penetración entre ellos.

La detección de colisiones aparece como una solución a problemas encontrados en diversas aplicaciones como la planificación del movimiento de robots, las animaciones basadas en la física (Lin \& Canny, 1992), el "rendering" háptico, el diseño y análisis en ingeniería basado en la simulación, la navegación en modelos virtuales con fines de entrenamiento en simuladores (Van Den Bergen, 1999) o en entretenimiento en video juegos, etc. Numerosas disciplinas han abordado problemas relacionados con la detección de colisiones como la robótica, la computación gráfica, la simulación de modelos físicos, el diseño asistido por computador (Haverkort, et al., 2002), la manufactura, la simulación molecular, la realidad virtual, así como en otros campos afines.

Para resolver el problema de la detección de colisiones se han propuesto una gran variedad de técnicas (García-Alonso, et al., 1994) tales como métodos analíticos, técnicas analíticas exactas, representaciones jerárquicas y métodos de optimización. Para modelos de objetos formados por caras poligonales es común utilizar detectores de colisión jerárquicos basados en volúmenes limitantes (Agarwal, 1997). Los volúmenes limitantes involucran métodos exactos y analíticos. Un volumen limitante es una primitiva sencilla que recubre generalmente un objeto ó conjunto de objetos. Las primitivas utilizadas como volúmenes limitantes deben de tener una prueba de intersección rápida. En definitiva, (Krishnan, et al., 1998), se trata de aproximar un objeto mediante volúmenes limitantes. De esta manera la colisión entre dos objetos se calcula mediante la intersección entre volúmenes limitantes. Si dos volúmenes limitantes no intersecan tampoco colisionan los objetos contenidos en los volúmenes limitantes. Los volúmenes limitantes sirven como prueba de nointersección.

En este artículo se muestra el desarrollo de un detector de colisiones jerárquico basado en una doble representación esférica. Se ha desarrollado un detector de colisiones jerárquico para poliedros convexos. Se han utilizado las esferas como volúmenes limitantes. Se considera tanto la esfera mínima que recubre un poliedro como la esfera máxima contenida en un poliedro. La esfera mínima representa el volumen limitante exterior y la esfera máxima representa el volumen limitante interior. La novedad del detector desarrollado es que i) la intersección entre las esferas externas cumple el papel de prueba de no intersección y ii) la intersección entre las esferas internas cumplen el papel de prueba de colisión, pues si dos esferas internas intersecan los poliedros que las contienen están en colisión.

Se realizan varios experimentos para estudiar el desempeño del detector de colisiones. En particular, se estudia la influencia de la esfera interior en la detección temprana de colisiones y se muestra que tiene un efecto significativo en la reducción del tiempo de ejecución de la detección de la colisión.

La organización del trabajo es la siguiente. La sección 1.1 describe los antecedentes del trabajo. El problema de la detección de colisiones es abordado en la sección 2 . Un poliedro convexo, su centroide y su orientación son descritos en la sección 3. En la sección 4 se desarrolla la representación jerárquica propuesta y el algoritmo del detector de colisiones. En la sección 5 se expone el proceso de triangulación de las caras de un poliedro convexo. En la sección 6 se describe el algoritmo de la intersección triángulo-triángulo utilizado. Los principales resultados del trabajo y 
la discusión sobre los resultados obtenidos se dan en la sección 7. Las principales conclusiones y los trabajos futuros se dan en las secciones 8 y 9 respectivamente. Finalmente, las referencias aparecen en la sección 10.

\subsection{Antecedentes del trabajo}

Se reconoce en la literatura la importancia que tienen las representaciones interiores de los objetos para detectar tempranamente la existencia de intersecciones, (Del Pobil \& Serna, 1995; Van Den Bergen 1997; Gottschalk, 1998). Sin embargo, la mayoría de implementaciones de detectores de colisiones basadas en las representaciones jerárquicas utilizan volúmenes limitantes exteriores que rápidamente puedan encontrar los pares de primitivas que no se intersecan. Son muy escasos los trabajos (Hubbard, 1996; Pitt-Francis \& Featherstone 1998; Arcila et al., 2004; Arcila, 2008) que utilizan representaciones interiores como parte de la jerarquía de volúmenes limitantes para acelerar la detección de la colisión. Las representaciones internas no han recibido la importancia que se merecen en la investigación debido, en parte, a la dificultad de aproximar por dentro los objetos geométricos.

La jerarquía de esferas propuestas por Del Pobil \& Serna (1995) es una de las pocas jerarquías que contienen tanto esferas exteriores como esferas interiores. Estas jerarquías se crean por separado haciendo que las pruebas de búsqueda de intersecciones se tornen complicadas.

\section{El problema de la detección de colisiones}

\subsection{Generalidades de la detección de colisiones}

La detección de colisiones es un problema fundamental en muchos aspectos de la computación geométrica y aparece en numerosas aplicaciones. Por ejemplo, en la animación de una escena es importante evitar colisiones entre los elementos de la misma Lin \& Canny (1992). En la planificación del movimiento de robots es indispensable un detector de colisiones que indiquen si el robot va a colisionar con los obstáculos del entorno. Decidir si dos cuerpos colisionan es un problema difícil. Sin embargo, decidir eficientemente si dos cuerpos colisionan sin importar la complejidad geométrica de los objetos es un reto (García-Alonso, et al., 1994).

\subsection{Algoritmos}

Los algoritmos de detección de colisiones tradicionalmente han requerido de una gran cantidad de pruebas de intersección, verificando si todos los polígonos o aristas que representan la superficie de un objeto intersecan algún polígono o arista del otro objeto. La mayoría de investigadores en el área proponen algoritmos que representen al objeto en una estructura jerárquica de primitivas sencillas de forma que se reduzca el número de llamadas para verificar la intersección entre los objetos. Afortunadamente, estos algoritmos han demostrado ser efectivos y en la mayoría de las aplicaciones solamente se verifica una pequeña fracción de pares de objetos. Dados dos o más objetos, la meta es verificar si existe una intersección entre ellos, si las estructuras jerárquicas presentan intersección, entonces se aplica una prueba de verificación más precisa. Las estructuras jerárquicas se dividen en dos grupos: la jerarquía de la subdivisión del espacio y la subdivisión del objeto. En la jerarquía de la subdivisión del espacio, los objetos se subdividen en una jerarquía espacial y son agrupados jerárquicamente según las regiones en las que se encuentran; de este modo, cuando un objeto cambia de posición, se establecen las nuevas regiones a las que pertenece y se detectan las intersecciones únicamente con los objetos que pertenezcan a esas regiones. Existen diversas maneras de subdividir el espacio, entre las más comunes están los Octrees, los Quadtrees, los árboles K-d y los árboles BSP (García-Alonso, et al, 1994). La subdivisión del objeto se trata en el siguiente apartado.

\subsection{Subdivisión jerárquica del objeto, volúmenes limitantes y árbol jerárquico}

En la jerarquía de la subdivisión del objeto, una escena se divide en jerarquías de volúmenes 
limitantes. Un volumen limitante es una primitiva sencilla que encierra uno o varios modelos de objeto (Krishnan, et al, 1998). Los volúmenes limitantes deben de tener una prueba de intersección rápida y deben aproximar lo más ajustadamente posible la geometría de un objeto por fuera si es un volumen exterior y por dentro si es un volumen interior. Para verificar la colisión entre dos objetos, es necesario verificar si las jerarquías de volúmenes limitantes de los objetos se intersecan. Los métodos basados en este tipo de aproximación incluyen el uso de esferas, cajas alineadas con los ejes (AABB), cajas orientadas (OBB), k-politopos de orientación discreta (kDOPs), cilindros, elipsoides (García-Alonso et al., 1994; Arcila et al., 2005). La clave es hacer que la prueba de intersección entre volúmenes limitantes sea menos costosa que la prueba de colisión entre objetos.

La jerarquía de representación de un objeto es un árbol de volúmenes limitantes que se calcula en un pre-procesamiento inicial. El volumen limitante en la raíz del árbol recubre el objeto original, los volúmenes limitantes de los nodos intermedios y terminales recubren partes del objeto original y todos los volúmenes limitantes en los nodos terminales reconstruyen el objeto original. Las pruebas de colisión entre dos objetos son realizadas entre sus árboles. Si el volumen limitante de la raíz de un árbol no se interseca con el volumen limitante de la raíz del otro árbol, los objetos no colisionan, de lo contrario, se debe bajar un nivel en los árboles y repetir la evaluación hasta el último nivel del árbol o hasta descartar la presencia de colisiones. Si los volúmenes limitantes de los nodos hijos se intersecan, se procede a la prueba de intersección entre los triángulos de las caras de los subpoliedros que formen parte de las caras de los poliedros originales. En general, las pruebas de intersección entre los volúmenes limitantes de los nodos no terminales sirven para garantizar la ausencia de colisión. Por el contrario, las pruebas de intersección entre las primitivas de los nodos terminales sirven para detectar la colisión entre los cuerpos, normalmente consisten en pruebas de intersección entre triángulos de la superficie de un objeto con triángulos de la superficie del otro objeto.

\subsection{Construcción del árbol jerárquico de la representación de un objeto}

Independientemente de la representación jerárquica utilizada hay dos formas de construir el árbol jerárquico de la representación: $i$ ) de arribaabajo y ii) de abajo-arriba. En el caso arriba-abajo se empieza asignando un volumen limitante a todo el objeto. Seguidamente el objeto se subdivide de acuerdo a una regla de subdivisión hasta un cierto nivel. En la Figura 1 se ilustra en dos-dimensiones un pentágono representado por (a) círculos exteriores y (b) círculos interiores, correspondientes al nivel 4. En la generación de los círculos exteriores, aquellos que se encuentran completamente en el polígono son marcados como interiores. En el caso de abajo-arriba se asignan volúmenes limitantes a las primitivas de los objetos, que normalmente son los triángulos de sus caras. Seguidamente, mediante una regla de combinación se llega a un volumen limitante que está en la raíz del árbol y que encierra todas las primitivas.

\section{Definición del poliedro, su centroide y su orientación}

\subsection{Definición de poliedro}

Un poliedro se define como la intersección de un número finito de semiplanos. En la práctica esto equivale al conjunto del espacio definido por el conjunto de desigualdades lineales de la ecuación (1).

$$
P=\left\{v \in \mathfrak{R}^{3}: M v \leq b\right\}
$$

Donde $M \in \mathfrak{R}^{m \times 3}$ es una matriz de rango 3. Existen diversas clasificaciones para los poliedros que dependen de las características geométricas, una de ellas es la división entre poliedros convexos y cóncavos. Se dice que un poliedro es convexo si para cualquier línea que se trace entre dos puntos interiores de un poliedro se genera un segmento de recta que interno al poliedro, de otro modo, si el segmento de línea se sale del interior del poliedro, se dice que es cóncavo. 


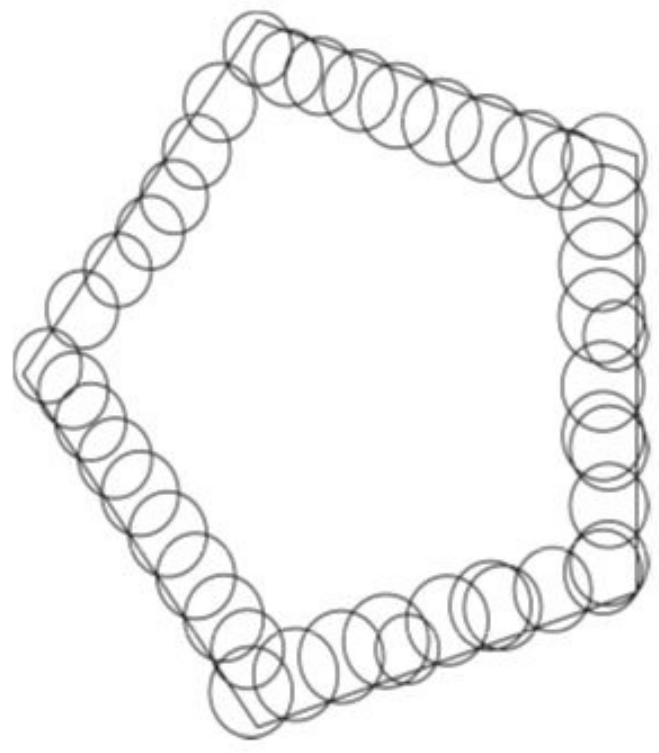

(a) Círculos Externos

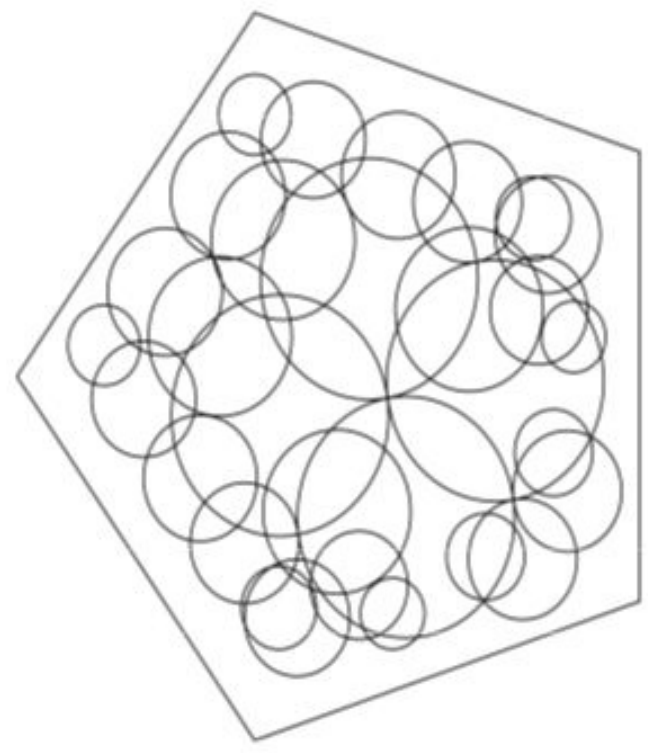

(b) Círculos Internos

Figura 1. (a) Círculos externos de un pentágono, (b) Círculos internos de un pentágono. Tanto los círculos internos como los externos hacen parte de la jerarquía de volúmenes limitantes para un poliedro.

\subsection{Centroide y orientación de un poliedro}

Se asume que las caras del poliedro están formadas por polígonos triangulados, por tanto, todos los polígonos están formados por triángulos. Sea $n$ el número total de triángulos de las caras del poliedro. Sean los puntos $p^{i}, q^{i} y r^{i}$ los vértices del $i$ ésimo triángulo, el centroide de un poliedro es su vector media $c e$, cuya definición esta en la ecuación(2):

$$
c e=\frac{1}{3 n} \sum\left(p^{i}+q^{i}+r^{i}\right)
$$

La orientación del poliedro se obtiene de la matriz de covarianza $\mathrm{C}_{v}$, la cual se define en la ecuación (3),

$$
C_{v\{j k\}}=\frac{1}{3 n} \sum \bar{p}_{j}^{i} \bar{p}_{k}^{i}+\bar{q}_{j}^{i} \bar{q}_{k}^{i}+\bar{r}_{j}^{i} \bar{r}_{k}^{i}, 1 \leq j, k<3
$$

donde: . Cada uno de ellos es un vector $3 \mathrm{x} 1$ y son los elementos de la matriz de covarianza de $3 \times 3$. La matriz de covarianza es una matriz simétrica positiva. Los vectores propios de $\mathrm{C}_{\mathrm{v}}$ normalizados forman una base ortonormal con la orientación del poliedro. Para crear la caja mínima que envuelve al poliedro y que tiene la orientación del poliedro, es necesario referir los vértices extremos del poliedro a la nueva base.

\section{EI Detector de colisiones propuesto}

\subsection{La representación doble de esferas}

La representación esférica propuesta es una generalización para poliedros convexos de la representación propuesta por (Arcila et al, 2002). La restricción de la representación a poliedros convexos no constituye una limitación importante debido a que todo poliedro no-convexo puede descomponerse como una unión finita de poliedros convexos.

La esfera es el volumen limitante más sencillo y frecuentemente se ha utilizado porque conduce a una prueba de intersección muy eficiente (Del Pobil \& Serna, 1995): basta comparar la distancia 
entre los centros de las esferas con la suma de sus radios. Esta prueba se muestra en el plano en la Figura 2. La Figura 2 (a) representa el caso en el que dos círculos están separados, es decir la distancia $d$ entre sus centros es mayor que la suma de sus radios; mientras que la Figura 2(b) representa el caso en el que los círculos se tocan, es decir la distancia d entre sus centros es menor que la suma los sus radios. Además la esfera es invariante frente a las rotaciones, lo cual permite una rápida actualización de la representación esférica. El inconveniente de las esferas es que no representan ajustadamente algunos objetos, en tal caso se requieren muchos niveles de subdivisión. Sin embargo, hay diferentes propuestas de árboles en la literatura. No todos ellos ajustan de la misma manera los objetos (Arcila, et al., 2004).

La representación esférica propuesta está formada por una jerarquía doble de esferas exteriores e interiores. Básicamente consiste en realizar una subdivisión binaria recursiva del poliedro de forma que se generen en cada subdivisión dos subpoliedros hijos. Por cada poliedro (o subpoliedro) que se genere en el árbol, se calculan dos esferas limitantes que deben ajustar al poliedro lo mejor posible: una exterior que aproxime por fuera al subpoliedro y una interior que aproxime por dentro al subpoliedro. La subdivisión se realiza hasta que se obtiene un árbol binario de una altura predeterminada que representa al poliedro con niveles adecuados para la detección de colisiones.

La raíz del árbol binario corresponde al poliedro original. En la raíz se almacena la esfera exterior que envuelve al poliedro y una esfera interior contenida en el poliedro. Se realiza una subdivisión binaria recursiva del poliedro de manera que en cada nodo del árbol se almacene un subpoliedro hijo junto con la esfera exterior e interior del subpoliedro. Este proceso se muestra en el plano en la Figura 3, en donde las esferas se remplazan por círculos y los poliedros por polígonos. En la Figura 3 se muestra un polígono $\mathrm{P}_{1}$ con su círculo exterior $\mathrm{C}_{\mathrm{Ext1}}$ y con su círculo interior $\mathrm{C}_{\text {Int }}$. Asimismo, se muestra el inicio de la subdivisión binaria. El polígono $\mathrm{P}_{1}$ se subdivide en dos polígonos hijos $\mathrm{P}_{1-1}$ y $\mathrm{P}_{1-2}$ mediante el eje de corte, el cual es perpendicular a la orientación del

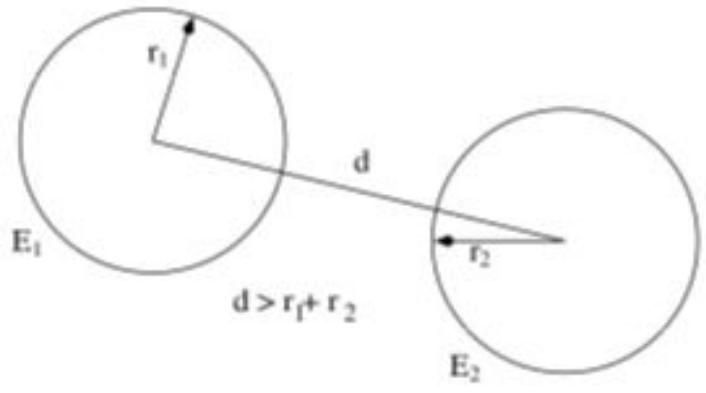

(a) ne intersección

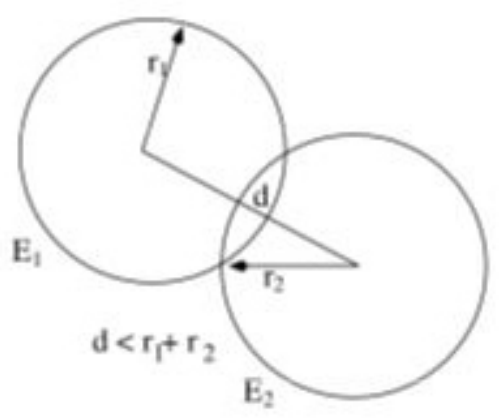

(b) intersección

Figura 2. Prueba de no Intersección y de intersección entre dos círculos.

polígono. La subdivisión se visualiza en la Figura 4 en la que se muestran los polígonos hijos $\mathrm{P}_{1-1}$ y $\mathrm{P}_{1}$. ${ }_{2}$ junto con sus círculos exteriores $\mathrm{C}_{\mathrm{Ext1-1}-1} \mathrm{y} \mathrm{C}_{\mathrm{Ext1-2}} \mathrm{y}$ sus círculos interiores $\mathrm{C}_{\text {Int1-1 }}$ y $\mathrm{C}_{\text {Int1-2. }}$. En la Figura 5 se muestran los nodos resultantes; el polígono original $\mathrm{P}_{1}$ junto a sus círculos envolvente $\mathrm{C}_{\mathrm{Ext1}} \mathrm{y}$ $\mathrm{C}_{\text {Int1 }}$ que forman la raíz del árbol binario de la subdivisión. Asi mismo los subpolígonos hijos $\mathrm{P}_{1}$. ${ }_{1} \mathrm{y}_{1-2}$ forman nodos hijos junto con el par de círculos envolventes exteriores e interiores $\mathrm{C}_{\mathrm{Ext1-1}}$, $\mathrm{C}_{\text {Int-1 } 1}, \mathrm{C}_{\text {Ext1-2 }} \mathrm{yC}_{\text {Int1-2 }}$ respectivamente. Finalmente, en los nodos terminales del árbol aparecen aquellas caras del subpoliedro padre que corresponden a partes de las caras exteriores del poliedro original. 


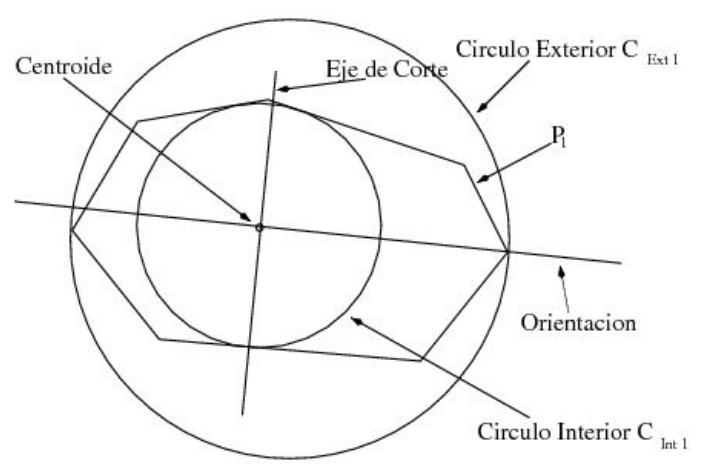

Figura 3. Para el Poliedro $P_{1}$ se define el centroide, el eje de corte, la orientación, los círculos interior $-C_{\text {Int } 1} y$ exterior - $C_{E x t l}$.

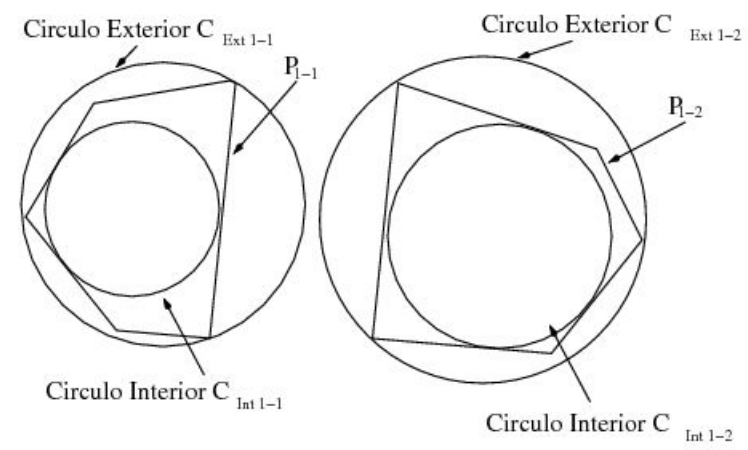

Figura 4. División del polígono $P_{1}$ en los polígonos $P_{1-1}$ y $P_{1-2}$

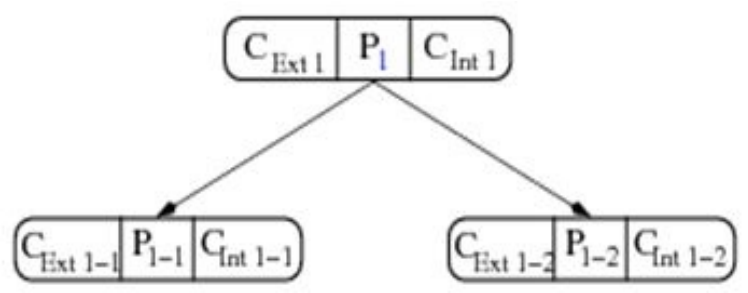

Figura 5. Estructura del nodo del árbol de la representación. Para cada uno de los nodos se almacena el polígono (centro), el círculo exterior (izquierda) y el círculo interior (derecha). La unión de los poligonos $P_{l-1}$ y $P_{l-2}$ reconstruyen el polígono $P_{1}$.
El cálculo de la esfera exterior de un poliedro se realiza buscando la esfera mínima que envuelve al poliedro. Se toman todos las conjuntos de 4 vértices del poliedro y se calcula el radio de la esfera; solo se consideran las esferas que envuelven todos los vértices del poliedro y de ellas se toma la esfera de radio mínimo. El cálculo de la esfera interior se realiza tomando como centro el centroide del poliedro; el cual, al igual que la orientación, se calcula a partir de la matriz de covarianza del poliedro. El radio de la esfera - interior es la menor de las distancias del centroide a las caras del poliedro. La subdivisión binaria se realiza con respecto a un plano que pasa por el centro de la esfera externa y perpendicular a la orientación del poliedro.

\subsection{El Algoritmo propuesto}

El objetivo de cualquier detector de colisiones jerárquico es el de rechazar rápidamente cualquier par de polígonos que no están en contacto. En este trabajo se presenta un esquema diferente; no solo se rechaza rápidamente cualquier par de polígonos separados sino que mediante el uso de las esferas internas, se detecta rápidamente cualquier par de polígonos que estén en contacto (Arcila, 2008).

Sean dos objetos poliédricos convexos $\mathrm{C}_{1}$ y $\mathrm{C}_{2}$. Sean $\mathrm{A}_{\mathrm{C} 1} \mathrm{y}_{\mathrm{C} 2}$ el árbol binario de esferas exteriores e interiores de $\mathrm{C}_{1}$ y $\mathrm{C}_{2}$ respectivamente. Si una esfera externa $E_{\text {Ext1 }}$ de $C_{1}$ no interseca la esfera externa $\mathrm{E}_{\mathrm{Ex} 12}$ de $\mathrm{C}_{2}$ se puede garantizar que no existe intersección entre los poliédricos $\mathrm{C}_{1} \mathrm{y}_{2}$ recubiertos por $\mathrm{E}_{\mathrm{Exxt}} \mathrm{y} \mathrm{E}_{\mathrm{Ex \times 2} 2}$. Esta es el prueba de rechazo rápido de cualquier detector de colisiones jerárquico. La prueba de aceptación rápida de la colisión por parte del detector que se desarrolla en este trabajo, se fundamenta en que si cualquier esfera interna $\mathrm{E}_{\text {Int1 }}$ de $\mathrm{C}_{1}$ que interseque cualquier esfera interna $\mathrm{E}_{\mathrm{In} 12}$ de $\mathrm{C}_{2}$ garantiza que la parte interna de $\mathrm{C}_{1}$ interseca la parte interna de $\mathrm{C}_{2} \mathrm{y}$ por tanto se ha detectado colisión entre los dos cuerpos. Estas pruebas se realizan en un recorrido simultáneo de las dos representaciones de $\mathrm{C}_{1} \mathrm{y} \mathrm{C}_{2}$. En el caso en que existen colisiones entre las esferas externas pero no en las internas, no se puede determinar si hay colisión, por lo tanto, se procede a verificar entre las caras poligonales de los objetos. 
A continuación se muestra el pseudocódigo del Algoritmo 1 propuesto. Sean $\mathrm{n}_{\mathrm{c} 1} \mathrm{y}_{\mathrm{c} 2}$ nodos de los árboles $\mathrm{A}_{\mathrm{C} 1} \quad \mathrm{y}_{\mathrm{C} 2}$, respectivamente. Sean las funciones: en_contacto $\left(E_{l}, E_{2}\right)$ para determinar si las esferas $\mathrm{E}_{1}$ y $\mathrm{E}_{2}$ se tocan, rec_ext (n) para obtener la esfera exterior del nodo $n_{c}$ de la representación de $\mathrm{C}$, rec_int (n) para obtener la esfera exterior del nodo $\mathrm{n}_{\mathrm{c}}$ de la representación de C, la función es_hoja $(n)$ determina si el nodo $\mathrm{n}_{\mathrm{c}}$ es una hoja del árbol $\mathrm{A}_{\mathrm{C}} \mathrm{e}$ hijos(n) retorna los hijos del nodo $n_{c}$.

\section{Algoritmo 1: Algoritmo General de Intersección}

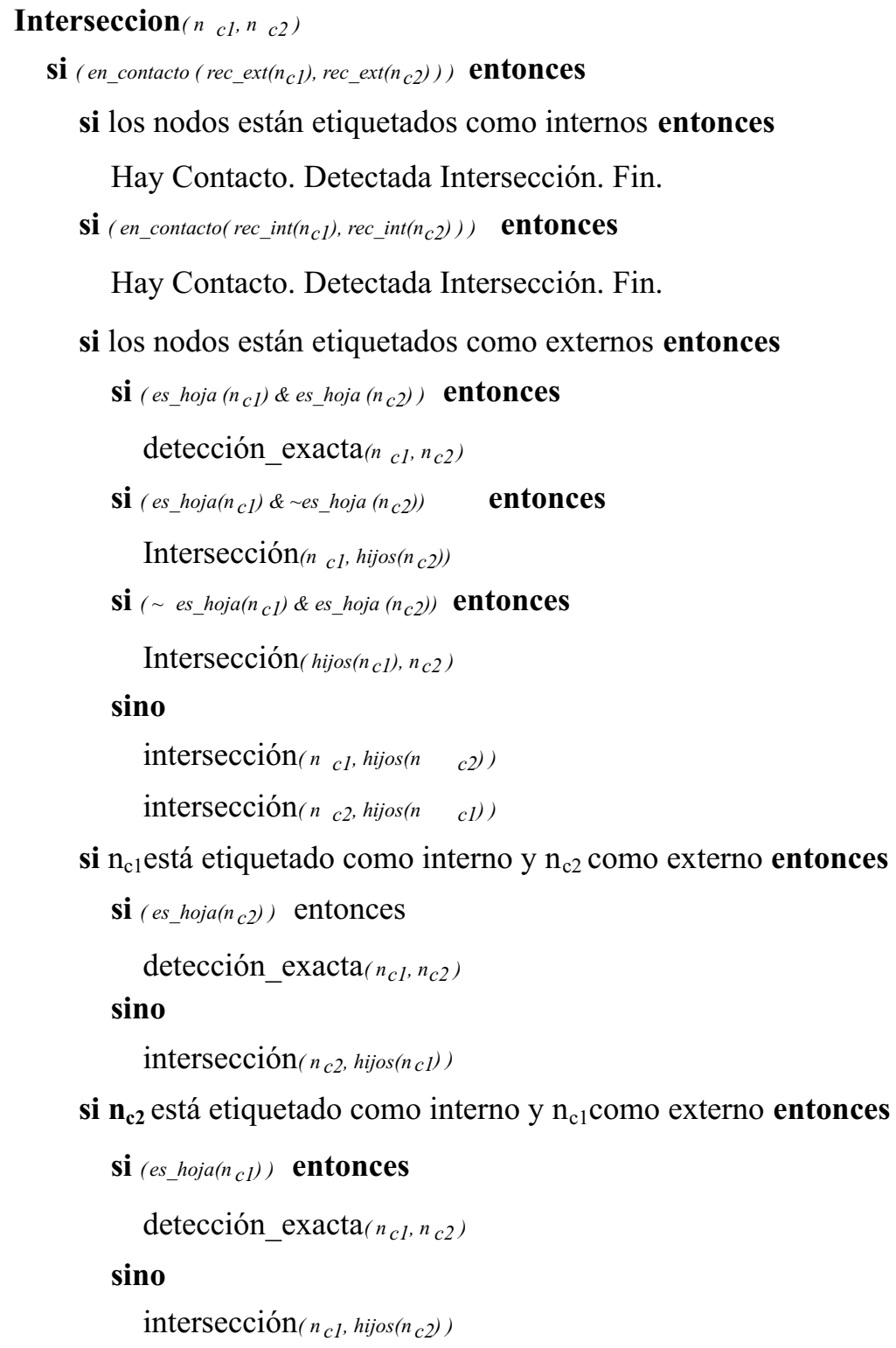

Si los nodos no se intersectan entonces

No hay contacto. Fin. 
La función detección_exacta $\left(n_{c 1}, n_{c 2}\right)$ calcula si hay intersección entre los pares de polígonos de las caras de los poliedros $\mathrm{C}_{1} \mathrm{y} \mathrm{C}_{2}$ que se encuentran asociados a los nodos hojas $n_{\mathrm{c} 1}$ y $\mathrm{n}_{\mathrm{c} 2}$ respectivamente.

\subsection{Esquema del algoritmo de intersección entre las caras poligonales.}

Cuando hay intersección y no se ha podido realizar por intermedio de las esferas interiores de las jerarquías, se llega a un par de nodos hojas que corresponden a partes de las caras externas de los subpoliedros. Entonces, se procede a verificar si las caras de los subpoliedros contenidos en las hojas intersecan o no. Estas caras externas están trianguladas, por lo que la intersección de cada par de caras se convierte en la búsqueda de intersecciones entre los triángulos que conforman las respectivas caras. Aquí es donde se hace intervenir el algoritmo de la intersección triángulo-triángulo de Moller (1997). Esta parte del algoritmo de detección de intersecciones hace que el algoritmo general sea exacto. A continuación se presenta el esquema del algoritmo de intersección entre las caras poligonales utilizado.

Sea caras_externas $\left(n_{c}\right)$ la función que retorna las caras externas del poliedro subdividido almacenado en el nodo $n_{c j} \operatorname{con} j=1,2$ de alguno de los árboles que intervienen en el proceso de detección de intersecciones. Sea la función triángulos $\left(n_{i j}\right)$ la función que retorna los triángulos en los cuales se descompuso la cara $n_{i j}$. Sea la función intersección_triángulo_triángulo $\left(t_{i j}, t_{k}\right)$ la función que determina si los triángulos $n_{i j}$ y $n_{k l} y$ se intersecan o no.

\section{Algoritmo 2: Algoritmo Intersección Exacta}

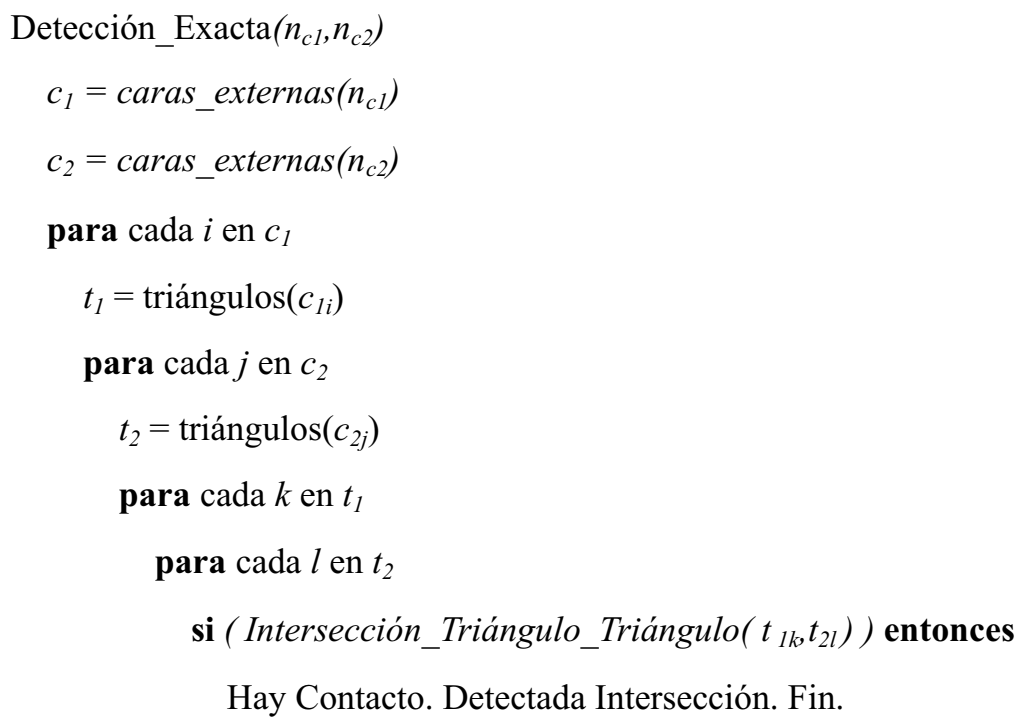

No hay Contacto. Fin.

\section{Resultados y discusión}

Se ha implementado el detector de colisiones jerárquico con esferas exteriores e interiores. $\mathrm{El}$ detector se ha realizado en $\mathrm{C}++$ usando el compilador g++ (GCC) 4.0.3. Los experimentos se han realizado sobre un computador con 4 procesadores Intel(R) Xeon(TM) CPU $2.80 \mathrm{GHz}$ sobre Linux Ubuntu 7.0.4 (dapper) y 4GB en la memoria RAM. 
Se han realizado varios experimentos con los poliedros convexos siguientes: a) cubo, b) octaedro, c) diamante, d) rh y e) troctaedro. La descripción de los poliedros se hace en términos de número de vértices, aristas y caras (Tabla 1) y en la Figura 6 se muestran los poliedros considerados. Las parejas de poliedros consideradas en los experimentos son: 1) troctaedro-diamante, 2) troctaedro-rh, 3) octaedro-diamante y 4) cubo-cubo (Arcila, 2008).

El espacio de experimentación considerado es una esfera en la que se coloca en su interior dos poliedros convexos. Con el detector se determina en cada ejecución si los objetos están en colisión o no hay colisión y seguidamente se mide el tiempo de respuesta. Cuando exista una colisión, se determina si ha sido detectada por una colisión esfera interna - esfera interna o debido a la intersección entre las caras de los poliedros. En total se efectúan 1.000.000 ejecuciones en las cuales se ubican los poliedros en posiciones y orientaciones aleatorias (Arvo, 1992) y se promedian los resultados. Esto se realiza para cada nivel del árbol binario de la representación. Se ha tomado el nivel 3 pues la experimentación muestra que es el nivel más significativo. Para cada colisión se han tomado dos tamaños del espacio de experimentación; un tamaño grande en el que la probabilidad de colisión es un $25 \%$ y un tamaño pequeño en el que la probabilidad de colisión es del $75 \%$.
Los resultados de las colisiones consideradas se dan en las Tablas 2 y 3 . La Tabla 3 compara el número de colisiones detectadas por intersección esfera interna - esfera interna frente al número de colisiones detectadas mediante la intersección entre las caras poligonales de los poliedros. Todo ello para los dos tamaños del espacio de experimentación. En la Tabla 2 se indican los tiempos empleados en los casos descritos en la Tabla 3.

El tiempo de detección de las colisiones mediante la intersección esfera interna-esfera interna es inferior al obtenido mediante la intersección caracara, como se indica en la tabla 2. Haciendo la comparación entre el tiempo y el número de intersecciones detectadas (ver tablas 2 y 3 ), se puede destacar el resultado obtenido con los polígonos Troctaedro y Rh. El tiempo usado para detectar 19067 intersecciones entre las esferas internas, es de $5.69 \mu \mathrm{seg}$, contrasta con el tiempo usado para detectar 6297 intersecciones entre las caras de los polígonos comparación: con el $375.18 \mu \mathrm{seg}$. En términos generales, $0.01 \%$ del tiempo fue empleado para decidir el $75 \%$ de las intersecciones entre los dos poliedros.

Comparando los resultados expuestos en la tabla 3 , en todas las colisiones consideradas se evidencia que el número de las colisiones detectadas mediante la intersección de esferas internas es superior al número de colisiones

Tabla 1. Tabla de poliedros considerados en los experimentos. Para cada uno se especifica el número de vértices, aristas y caras.

\begin{tabular}{lccc}
\hline \multicolumn{1}{l}{ Nombre Cuerpo } & No. Vértices & No. Aristas & No. Caras \\
\hline Cubo & 8 & 12 & 6 \\
Octaedro & 6 & 12 & 49 \\
Diamante & 41 & 88 & 62 \\
Rh & 120 & 180 & 14 \\
Troctaedro & 24 & 36 & \\
\hline
\end{tabular}




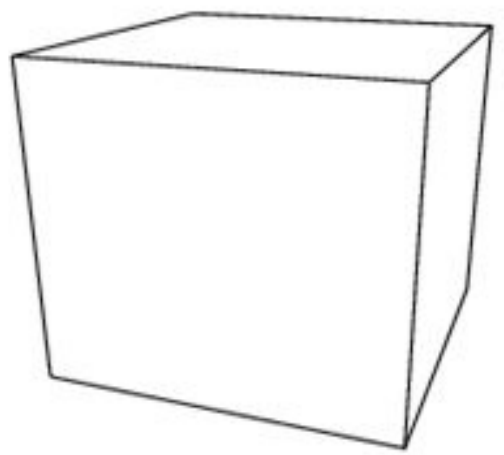

(a) Cubo

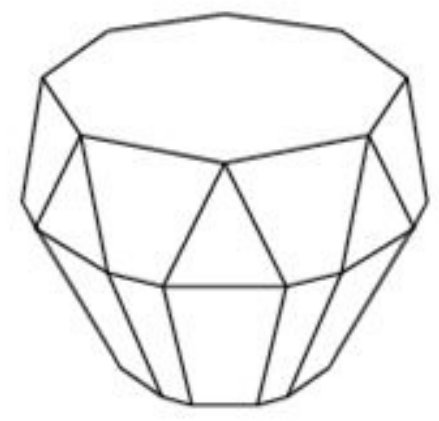

(c) Diamante

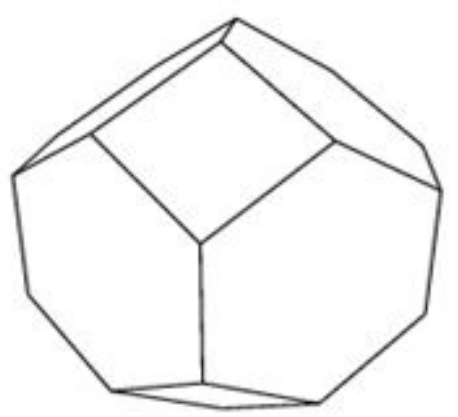

(e) Troctaedro

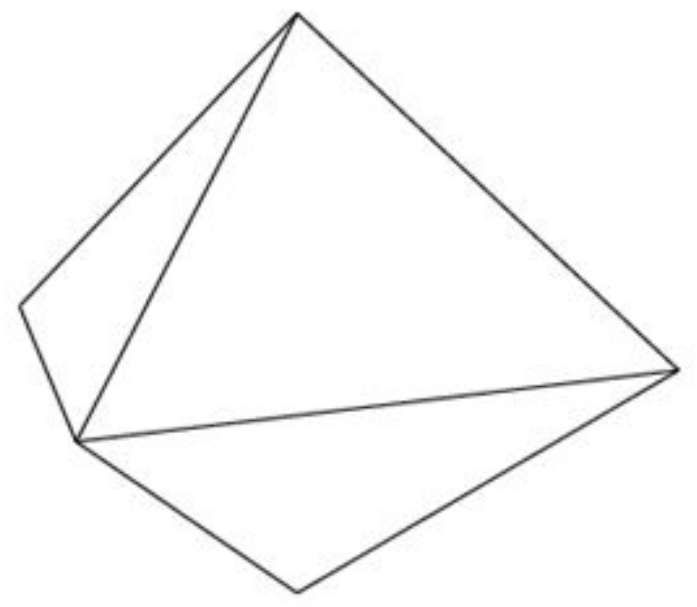

(b) Octaedro

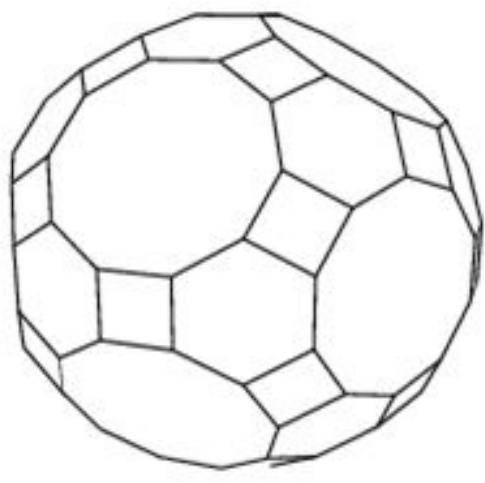

(d) $R h$

Figura 6. Poliedros considerados en el experimento. 
Tabla 2. Tiempo (useg) empleado en detectar las colisiones. El tiempo se calcula para dos espacios de experimentación propuestos de acuerdo a la probabilidad de colisión. Se evalúan los tiempos empleados en las intersecciones entre las esfera internas y entre las caras de los poliedros.

Tamaños del Espacio de Experimentación

(25\%) Tamaño Grande

(75\%) Tamaño Pequeño

\begin{tabular}{lcccc}
\hline & $\begin{array}{c}\text { Tiempo }(\mu \mathrm{seg}) \text { de } \\
\text { intersecciones entre } \\
\text { esferas internas }\end{array}$ & $\begin{array}{c}\text { Tiempo }(\mu \mathrm{seg}) \text { de } \\
\text { intersecciones entre } \\
\text { caras de los poliedros }\end{array}$ & $\begin{array}{c}\text { Tiempo }(\mu \mathrm{seg}) \text { de } \\
\text { intersecciones entre } \\
\text { esferas internas }\end{array}$ & $\begin{array}{c}\text { Tiempo }(\mu \mathrm{seg}) \mathrm{de} \\
\text { intersecciones entre } \\
\text { caras de los poliedros }\end{array}$ \\
Troctaedro - Diamante & 3.99 & 127.62 & 3.07 & 118.13 \\
Troctaedro - Rh & 5.69 & 375.18 & 3.86 & 379.59 \\
Octaedro - Diamante & 1.83 & 45.50 & 1.65 & 42.73 \\
Cubo - Cubo & 3.91 & 12.70 & 2.85 & 13.29 \\
\hline
\end{tabular}

Tabla 3. Número de colisiones detectadas. La cantidad de colisiones se calcula para dos espacios de experimentación propuestos de acuerdo a la probabilidad de colisión. Se evalúa la cantidad de intersecciones entre las esfera internas y entre las caras de los poliedros.

Tamaños del Espacio de Experimentación
(25\%) Tamaño Grande
(75\%) Tamaño Pequeño

\begin{tabular}{|c|c|c|c|c|}
\hline & $\begin{array}{l}\text { Número de } \\
\text { Intersecciones entre } \\
\text { esferas internas }\end{array}$ & $\begin{array}{c}\text { Número de } \\
\text { Intersecciones entre } \\
\text { caras de los poliedros }\end{array}$ & $\begin{array}{l}\text { Número de } \\
\text { Intersecciones entre } \\
\text { esferas internas }\end{array}$ & $\begin{array}{c}\text { Número de } \\
\text { Intersecciones entre } \\
\text { caras de los poliedros }\end{array}$ \\
\hline Troctaedro - Diamante & 15229 & 10273 & 54842 & 21803 \\
\hline Troctaedro - Rh & 19067 & 6297 & 62718 & 12359 \\
\hline Octaedro - Diamante & 15984 & 10384 & 55204 & 21167 \\
\hline Cubo - Cubo & 15859 & 12310 & 51688 & 24735 \\
\hline
\end{tabular}

detectadas mediante la intersección entre las caras poligonales de los poliedros. Esto se debe a que las pruebas realizadas con los polígonos internos permite identificar de inmediato la colisión entre los polígonos y se hace innecesario en algunos casos llegar hasta el nivel de la comparación de las caras poligonales que es computacionalmente más costosa. En general, hay un factor de dos o tres veces superior al número de las colisiones que se obtienen mediante las esferas internas. Es decir que no solo las esferas internas contribuyen a la detección de colisiones sino que también contribuyen a disminuir el tiempo de la detección.

\section{Conclusiones}

Se ha diseñado una nueva representación geométrica jerárquica doble basada en esferas exteriores e interiores. Con la representación doble se ha implementado un algoritmo de detección de colisiones jerárquico para poliedros convexos. Es de destacar, la enorme importancia que juegan las esferas interiores en la detección de colisiones. Las intersecciones esfera interna esfera interna son por lo general no solo más numerosas que las intersecciones entre las caras de los poliedros sino que también son más rápidas. 
La novedad del detector implementado consiste en que la representación jerárquica no solo juega el papel de prueba de no-intersección, como en los detectores tradicionales, sino también de prueba de aceptación de la colisión. La incorporación de una representación doble contribuye al desempeño de los detectores de colisión. Esta forma de proceder está de acuerdo con la idea de la introducción de los volúmenes limitantes en los detectores de colisión consistente en detectar la mayor parte de las colisiones antes de llegar a los nodos de los árboles de las representaciones.

\section{Referencias bibliográficas}

Agarwal, P. K. (1997). CRC Handbook of Computational Geometry, In J. E. Goodman and J. O'Rourke (eds.), CRC Press. p. 575-598.

Arcila. (2008). Nuevas Representaciones Dobles (Externas e Internas) en Detectores de Colisiones Jerarquicos. Doctoral Thesis, Facultad de Ingeniera, Universidad del Valle. Cali.

Arcila, O., \& Bañón, J. M. (2005). Un Nuevo Detector de Colisiones basado en Elipsoides. In Memorias de CLEI XXXI, Cali, Colombia.

Arcila, O., Bañón, J. M., \& Muñoz, C. A. (2004). Un Modelo de Representación Circular Para Polígonos. Energía y Computación. XI(2), p. 6-13.

Arcila, O., Muñoz, C. A., \& Bañón, J. M. (2002). Un Algoritmo Eficiente para la Detección de Colisiones en Tiempo Real. In Memorias de CLEI XXVIII, Montevideo, Uruguay.

Arvo, J. (1992). Graphics Gem III. Academic Press.
Del Pobil, A. P., \& Serna, M. A. (1995). Spatial Representation and Motion Planning, Heidelberg:Springer-Verlag.

García-Alonso, A., Serrano, N., Flaquer, J. (1994). Solving the collision detection problem. IEEE Computer Graphics and Applications, 13(3): p. 36-43,

Gottschalk, S. (1998). Collision Queries using Oriented Bounding Boxes. Doctoral Thesis, Department of Computer Science, University of North Carolina at Chapell Hill.

Haverkort, H. J., de Berg, M., \& Gudmundsson, J. (2002). Box-trees for collision checking in industrial installations. In Proceedings 18th Symp. Computational Geometry (SoCG). p. 53-62.

Hubbard, P. M. (1996). Approximating polyhedra with spheres for time-critical collision detection, ACM Transactions on Graphics, 15 (3), p. 179-210.

Krishnan, S., Pattekar, A., Lin, M., \& Manocha, D. (1998). Spherical shell: a higher order bounding volume for fast proximity queries. In Proceedings WAFR'98.

Lin, M., \& Canny, J. (1992). "Efficient collision detection for animation." In Third Eurographics Workshop on Animation and Simulation, Cambridge, England, September.

Moller, T., (1997). A Fast Triangle-Triangle Intersection Test, Journal of Graphics Tools, 2(2), p 25-30.

Pitt-Francis, J., \& Featherstone, R. (1998). Automatic Generation of Sphere Herarchies from CAD Data. In Proceedings of the IEEE International Conference on Robotics and Automation. p 324-329. 
Van Den Bergen, G. J. A. (1997). Efficient Collision Detection of Complex Deformable Models using AABB Trees, Journal of Graphics Tools: JGT, 2(4), 1-14.

Van Den Bergen, G. J. A. (1999). Collision Detection in Interactive $3 D$ Computer Animation. Doctoral Thesis, Eindhoven Technical University. 